\title{
Return to sport following anterior cruciate ligament reconstruction
}

\author{
Julian Feller • Kate E. Webster
}

Received: 1 October 2012 /Accepted: 14 October 2012/Published online: 10 November 2012

(C) Springer-Verlag Berlin Heidelberg 2012

\begin{abstract}
Rates of return to pre-injury sport following anterior cruciate ligament (ACL) reconstruction are less than might be expected from standard outcome measures and there appears to be a rapid decline in sporting participation after two to three years. There are many factors that influence whether an individual will return to sport following this type of surgery. They include not only surgical details and rehabilitation, but also social and psychological factors, as well as demographic characteristics. Age is of particular importance with older patients being less likely to resume their pre-injury sport. It is important that future research clearly identify the pre-injury characteristics of the study cohort when investigating return to sport, and also that there is consistent and precise terminology used to report rates of return to sporting activities. Little is known about how to determine when it is safe to return to sport following ACL reconstruction or how to predict whether an athlete will be able to successfully return to sport. Finally, it needs to be recognised that return to sport following ACL reconstruction is associated with a risk of further injury and the development of osteoarthritis.
\end{abstract}

\section{Discussion}

The aim of most people undergoing an anterior cruciate ligament (ACL) reconstruction is to return to some level of sporting activity, however modest. Despite many reports of successful outcomes following ACL reconstruction, recent reports have suggested that the rates of return to sport may

J. Feller $(\bowtie)$

OrthoSport Victoria,

Richmond, VIC, Australia

e-mail: jfeller@osv.com.au

K. E. Webster

La Trobe University,

Melbourne, Australia not match the success of this surgery as measured by other factors $[2,4,5,10,13,19,25,31,44]$. This discrepancy raises many issues and the purpose of this article is to explore return to sport following ACL reconstruction from a number of perspectives.

One of the most fundamental questions is what constitutes a return to sport? Precise terminology is important. A return to pre-injury sport may well be different to a return to pre-operative sport or sports activity level, particularly in the setting of chronic ACL insufficiency in which patients may well have participated in sport at a lower level or lesser intensity than prior to their ACL injury. In the past there may have been a tendency to use these terms interchangeably, giving an artificially high rate of return to the level of sport played prior to injury [1, 14].

Furthermore, although following an ACL reconstruction, a patient may return to the sport in which they previously participated, it is important to clarify whether they have returned to training or competition and, if competition, whether it is at the same level of competition as prior to injury. Even if they have returned to the same level of competition, it is another matter as to whether they play with the same competency. Sports activity levels may appear to provide an objective measurement of sporting participating levels, but there may still be a discrepancy between the sports activity level and participation in pre-injury sport $[15,31]$.

This of course assumes that, following surgery, the individual wishes to return to the same sport and at the same level. There are many factors that may influence patients' expectations and their post-operative expectations may not mirror their pre-operative expectations. Age appears to be an important factor. Older patients may not be as likely to return to their pre-injury sport as younger patients [2]. There may be many reasons for this. Work and family commitments may influence individuals' priorities, as may their motivation and abilityperceived or real-to recover from surgery and resume their previous sport. 
In assessing reported rates of return to sport it is therefore important to know about many aspects of the study cohort: age as mentioned above, gender, type of pre-injury sport and the level of participation, and patient expectations. Study groups are often heterogeneous, making it difficult to extrapolate the results to a specific group [47]. Elite sportspeople, although facing potentially greater challenges in resuming their sport at the same level, may have a greater motivation and greater support to achieve a successful return to sport $[30,46]$.

So what is the current reported rate of return to sport following ACL reconstruction? In a recent systematic review, Ardern et al. evaluated 48 studies that met their inclusion criteria [5]. The studies reported on outcomes in 5,770 patients at a mean follow-up of 42 months. Overall, $82 \%$ of patients returned to some kind of sport, but only $63 \%$ were participating in their pre-injury sport at followup. When competitive sport was considered, only $44 \%$ were participating at follow-up. These participation rates were in contrast to the findings that approximately $90 \%$ of patients were rated normal or nearly normal on impairment-based outcomes such as strength and knee laxity, and that $85 \%$ were rated as normal or nearly normal on the basis of activity outcome measures such as the International Knee Documentation Committee knee evaluation form.

These rates of return to sport are similar to subsequent reports. Mascarenhas et al. found that $50 \%$ of young ( 25 years or less at the time of ACL reconstruction) athletes returned to their pre-injury sport at some stage following surgery [31]. Brophy et al. reported that at a mean follow-up of seven years $36 \%$ of soccer players were still playing compared to the $72 \%$ that had resumed play at some time following their ACL reconstruction [10]. Similarly, Struewer et al. reported that the participation rate in pivoting and contact sports decreased from $78 \%$ at two years to $45 \%$ at 13.5 years, and for competitive sport from $65 \%$ to $19 \%$ [44]. Ardern et al. also found a discrepancy between the highest level of sport played at any time following surgery and sports participation at follow-up [2]. Sixty-one per cent had attempted their pre-injury sport at some time following ACL reconstruction, compared to $45 \%$ who were participating in their pre-injury sport at follow-up at a mean of 39 months. For any level of sports participation the numbers were 93 and $66 \%$, respectively.

These studies raise the question of at what point one assesses whether a return to sport has been achieved. Ardern et al. also reported on return to sport rates at 12 months following surgery and found those who had not attempted their pre-injury sport at 12 months were just as likely to have returned to their pre-injury sport at a mean of 39 months after their surgery [2]. In a separate study of patients who had participated in competitive pivoting sports prior to injury, the same authors found that although only $33 \%$ had attempted competitive sport at 12 months following ACL reconstruction, another $32 \%$ still intended to return to competitive sport [4].

Thus, assessing return to sport rates at 12 months may be too early and assessment of participation at three or more years may suggest lower "success" rates than considering the highest level of sport played at any time following ACL reconstruction surgery. In their systematic review, Ardern et al. found a slightly but statistically significantly higher rate of reported return to sport in studies with a mean follow-up of less than 24 months compared to those with a mean follow-up rate of 24 months or greater [5]. These findings seem to imply that there may be quite a rapid decline in sports participation after a peak at somewhere around two years following surgery. Patients who have successfully returned to sports may subsequently give up that sport or reduce their participation to a lower level for a variety of reasons [34, 37, 43, 44].

Although authors have frequently reported absolute rates of return to sport following ACL reconstruction, albeit at different follow-up times, there is less information about when the subjects actually resumed sport. Although the mean times to return to pre-injury sport may be relatively short - in the order of six months or less - there is typically a wide range of values reported. For instance, although the mean times to resume pre-injury sport were 3.8 and 6.4 months for double-bundle and single-bundle ACL reconstruction in a study by Zaffagnini et al., the range was three to 24 months [52]. Similarly, Shelbourne and Gray reported a mean time to return to competition at full capability of 6.2 months, but a range of two to18 months [40]. In both studies the patients participated in varying levels of sport, competitive and recreational, making it difficult to extrapolate from the results.

In assessing reported outcomes of ACL reconstruction it is also worth noting that clearance to return to sport at a certain time point does not necessarily mean that patients actually returned to sport at that time. For instance, if individuals play a sport that is played on a seasonal basis, the timing of their surgery with respect to the stage of the season may influence their return to participation. There may be an added incentive to return earlier, e.g. at the start of the next season, than for a sport which is played all year round [4]. Alternatively, return to sport may be delayed until the commencement of the next season despite the patient being cleared by the treating practitioner to resume sport.

Warner et al. attempted to identify literature that compared return to sport rates for different sports [47]. However, they found only minimal data and could only identify eight studies that met their inclusion criteria. Most of these studies considered only one sport and useful comparisons between individual studies were limited due to methodological differences. Once again they noted heterogeneous populations 
in terms of age, level of pre-injury participation, time of follow-up and definitions of return to sport. They were able to conclude that return to activities such as bicycling and jogging was more likely than to cutting and pivoting sports.

Clearly there are many factors that influence an individual's ability, desire and decision whether or not to return to sport and at what level. Dunn et al. tried to quantify some of these factors [13]. They found that a higher pre-injury activity level was the strongest predictor of return to sport at two years after ACL reconstruction, while female gender, a higher body mass index (BMI) and smoking in the first six months prior to surgery were associated with a lower rate of return to sport. Other authors have also identified gender as important, although the differences may disappear with longer follow-up [2, 4, 48].

Using the Tampa Scale of Kinesiophobia, Kvist et al. identified fear of re-injury as a significant factor in patients who did not return to their pre-injury activity level compared to those who did [23]. In another study to investigate the role of psychological factors in patients recovering from ACL reconstruction, Langford et al. [24] utilised the ACL Return to Sport after Injury scale (ACL-RSI scale) [49] and found that patients who returned to competitive sport at 12 months scored significantly higher (reflecting a more positive psychological response) at both six and 12 months than patients who did not return to competitive sport. More recently, Ardern et al. reported that patients who returned to their pre-injury sports level demonstrated less fear of reinjury than those who did not return to their pre-injury level [3]. Interestingly, for some of the questions used in their survey, patients who had surgery more than three months after injury had a greater fear of re-injury than those who had their surgery closer to the time of injury. One might speculate that this may reflect the patients having experienced episodes of giving way of their knee other than at the time of their original injury.

Apart from demographic, environmental and psychological factors, there are a number of surgical and rehabilitation variables that may also play a role in whether a patient returns to sports following ACL reconstruction. Most studies implicitly assume that the surgery has been done well from a technical perspective, although little is done to confirm this apart from measuring knee laxity. Graft types have often been compared, particularly hamstring tendon and patellar tendon autografts. No difference in rates of return to pre-injury activity level have been identified in either individual studies or meta-analyses [8, 19, 29, 31]. Similarly, no difference in return to sport rates has been identified between patellar tendon autografts and allografts [21].

Although there has been much discussion regarding tunnel position and graft orientation, particularly in recent years, there is little information about the impact of these surgical variables on return to sport. Although an earlier meta-analysis did not find a clinical difference between double-bundle and single-bundle ACL reconstructions in terms of knee laxity [32], more recent meta-analyses have shown improved joint stability with double-bundle procedures $[26,53]$. Better control of tibial rotation might be expected to give a better functional outcome and therefore a potentially higher rate of return to pre-injury activity, but to date, so-called anatomical reconstruction has not been shown to result in higher activity levels [53].

It has previously been noted that rates of return to preinjury sport might not be as high as would be expected on the basis of measurements of strength and knee laxity or activity outcome measures $[5,19]$. From a broader perspective, the lack of correlation between what might be regarded as standard outcome measures and rates of return to sport raises questions about what we should be measuring following ACL reconstruction and whether the current assessment tools address the appropriate issues or are limited by a ceiling effect. For instance, in the widely used Tegner scale [45], level 9 [competitive sports - soccer, football, rugby (lower divisions), ice hockey, wrestling, gymnastics, basketball] covers multiple levels of competition and participation, and it is quite possible for individuals to return to a lower level of their pre-injury sport but to maintain the same Tegner rating of 9. It is only if they drop from national representation (level 10) that a reduction will be identified.

Much has been written about rehabilitation protocols for patients who have undergone ACL reconstruction [11], but there has been little evaluation of the influence of these protocols on outcome. In their landmark paper, Shelbourne and Nitz described their accelerated rehabilitation protocol in patients who had an ACL reconstruction using a patellar tendon autograft [41]. They observed that patients who were non-compliant with earlier restrictive rehabilitation protocols, and who progressed as they felt comfortable, did in fact progress more rapidly, and regained their function more quickly, without any deleterious effects. In a subsequent randomised controlled trial, Beynnon et al. compared accelerated and non-accelerated rehabilitation protocols, again in patients who had undergone ACL reconstruction with a patellar tendon graft [7]. Although they did not investigate return to sport per se, Tegner activity levels did not differ between the two groups and no deleterious effects of the accelerated protocol were identified. Although widely used, the effect of an accelerated rehabilitation protocol in patients who have had an ACL reconstruction with a hamstring tendon graft has not been evaluated in a controlled fashion. Some case series have shown that accelerated rehabilitation is not detrimental to the outcome of hamstring tendon ACL reconstruction [28, 30], but Fujimoto et al. suggested that an early return to activity was associated with increased graft laxity [17]. 
Similarly, although there are many opinions about the criteria that should be met before an athlete resumes athletic participation following ACL reconstruction [11, 22, 33], there has been little prospective investigation of the validity of such criteria. In evaluating such criteria, one has to first determine the purpose of the criteria. Are they designed to determine whether an athlete is capable of resuming sport or are they designed to determine whether it is safe for an athlete to resume sport? In either case, one would need to record the outcomes of athletes who have and have not met the criteria and whether their ability to resume sport, either effectively or safely, reflects having or having not met the various criteria. To date, such an evaluation is lacking.

Returning to sport following an ACL reconstruction puts the individual at risk of both ACL graft rupture and rupture of the contralateral ACL. In a systematic review Wright et al. reported an overall risk of graft rupture of $5.8 \%$ at a minimum of five years follow-up and an overall rate of contralateral ACL rupture of $11.8 \%$ [51]. There was, however, a considerable range for both values. For ACL graft rupture the reported rates ranged from 1.8 to $10.4 \%$, and for contralateral ACL rupture, from 8.2 to $16 \%$.

Various factors have been associated with an increased risk of ACL graft or contralateral ACL rupture, but the evidence is conflicting with some authors finding few, if any, identifiable risk factors [37]. Shelbourne et al. found that a young age increased the risk of ACL graft rupture [42], whilst Bourke et al. did not find an effect of age, but did find that male gender and a positive family history of ACL injury were associated with an increased risk of ACL graft rupture [9]. It is not clear whether it was youth per se that was associated with an increased risk in the study by Shelbourne et al. or whether youth was a surrogate for other factors such as increased activity, increased risk-taking or incomplete neuromuscular development. With regard to contralateral ACL rupture, female gender [42] and a positive family history, a return to pre-injury sport and an ACL reconstruction with a patellar graft [9] have been identified as risk factors, although there are also studies that did not identify gender as a risk factor $[37,50]$. One study identified a young age as a important risk for contralateral ACL rupture, despite it not being a risk factor for ACL graft rupture in that particular series [20]. Overall, the variability between studies in terms of risk factors means that further investigation is still required.

Whilst Bourke et al. did not find a difference in graft rupture rates between patellar tendon and hamstring tendon grafts, allograft patellar tendon grafts have been associated with higher failure rates than autogenous patellar tendon grafts, particularly in young and active patients $[6,21]$.

Whilst it is often assumed that an early return to sport is associated with an increased risk of graft rupture, Shelbourne et al. found no difference in graft rupture rates in patients who returned to sport before and after six months following surgery [42]. All patients in this large cohort had a reconstruction with a patellar tendon autograft. Glasgow et al. had previously drawn similar conclusions, again in patients with patellar tendon grafts, but in a smaller cohort [18].

On the other hand, animal studies suggest that the graft goes through a maturation process that involves a number of stages that may put the graft at risk [11, 12, 39]. An initial period of necrosis and hypocellularity is followed by a phase of cellular proliferation and revascularisation. It is during that latter phase that the mechanical properties of the graft are at their lowest. Subsequently, a remodelling process, referred to as ligamentisation, occurs and the graft gradually adopts the morphology and mechanical properties of the native ACL. On this basis, a graft would be expected to be at increased risk of injury between six weeks and three months, a time period that corresponds to an increase in the demand of activities in most rehabilitation protocols. However, the mismatch between findings from animal studies and the limited evidence available from human grafts has been noted $[12,39]$. It appears that in humans tendon autografts survive in the intra-articular environment and may therefore be less susceptible to damage.

Another risk of returning to sport following ACL reconstruction is that of sustaining damage to the menisci or articular surfaces, or of aggravating damage sustained at the time of ACL rupture. This in turn may increase the risk of developing osteoarthritis. In the past, a high prevalence of osteoarthritis has often been cited for ACL deficient individuals. However, a more recent systematic review, which took into account the methodological quality of studies, reported that the prevalence of osteoarthritis is lower than widely quoted [36]. The authors found a prevalence of knee osteoarthritis at ten years between 0 and $13 \%$ for isolated ACL ruptures and between 21 and $48 \%$ for combined injuries. Intuitively one might expect that ACL reconstruction would reduce the risk of further injuries and perhaps reduce the risk of osteoarthritis compared to resuming sport with an ACL deficient knee. Although there is some evidence to support this, there is stronger evidence to suggest that ACL reconstruction does little to reduce the risk of subsequent osteoarthritis [16, 27, 35, 36, 38].

So one could perhaps look at the question of return to sport following ACL reconstruction from a philosophical perspective. That is, even if the surgery produces a wellfunctioning and stable knee, should athletes consider not returning to sport in an attempt to protect their knee from further injury and long-term degeneration? Such a view has been espoused [34]. This, however, begs the question of why reconstruct the ACL in the first place. With little evidence to show a protective effect of ACL reconstruction 
in terms of osteoarthritis, ACL deficient patients who do not wish to resume pivoting sports might well consider nonoperative management of their injury, unless instability interferes with activities of daily living.

\section{Summary}

In summary, the rates of return to pre-injury sport following ACL reconstruction are less than might be expected from standard outcome measures and there appears to be a rapid decline in sporting participation after two to three years. There are many factors that influence whether an individual will return to sport following this type of surgery. They include not only surgical details and rehabilitation, but also social and psychological factors, as well as demographic characteristics. Age is of particular importance with older patients being less likely to resume their pre-injury sport. It is important that future research clearly identifies the pre-injury characteristics of the study cohort when investigating return to sport, and also that there is consistent and precise terminology used to report rates of return to sporting activities. Little is known about how to determine when it is safe to return to sport following ACL reconstruction or how to predict whether an athlete will be able to successfully return to sport. Finally, it needs to be recognised by the practitioner and patient alike that return to sport following ACL reconstruction is associated with a risk of further injury and the development of osteoarthritis.

\section{References}

1. Anderson AF, Snyder RB, Lipscomb AB (2001) Anterior cruciate ligament reconstruction. A prospective randomized study of three surgical methods. Am J Sports Med 29:272-279

2. Ardern CL, Taylor NF, Feller JA, Webster KE (2012) Return-tosport outcomes at 2 to 7 years after anterior cruciate ligament reconstruction surgery. Am J Sports Med 40:41-48

3. Ardern CL, Taylor NF, Feller JA, Webster KE (2012) Fear of reinjury in people who have returned to sport following anterior cruciate ligament reconstruction surgery. J Sci Med Sport. doi:10.1016/j.jsams.2012.03.015

4. Ardern CL, Webster KE, Taylor NF, Feller JA (2011) Return to the preinjury level of competitive sport after anterior cruciate ligament reconstruction surgery: two-thirds of patients have not returned by 12 months after surgery. Am J Sports Med 39:538-543

5. Ardern CL, Webster KE, Taylor NF, Feller JA (2011) Return to sport following anterior cruciate ligament reconstruction surgery: a systematic review and meta-analysis of the state of play. $\mathrm{Br} \mathrm{J}$ Sports Med 45:596-606

6. Barrett GR, Luber K, Replogle WH, Manley JL (2010) Allograft anterior cruciate ligament reconstruction in the young, active patient: Tegner activity level and failure rate. Arthroscopy 26:1593-1601

7. Beynnon BD, Uh BS, Johnson RJ, Abate JA, Nichols CE, Fleming $\mathrm{BC}$ et al (2005) Rehabilitation after anterior cruciate ligament reconstruction: a prospective, randomized, double-blind comparison of programs administered over 2 different time intervals. Am J Sports Med 33:347-359
8. Biau DJ, Tournoux C, Katsahian S, Schranz P, Nizard R (2007) ACL reconstruction: a meta-analysis of functional scores. Clin Orthop Relat Res 458:180-187

9. Bourke HE, Salmon LJ, Waller A, Patterson V, Pinczewski LA (2012) Survival of the anterior cruciate ligament graft and the contralateral ACL at a minimum of 15 years. Am J Sports Med 40:1985-1992

10. Brophy RH, Schmitz L, Wright RW, Dunn WR, Parker RD, Andrish JT et al (2012) Return to play and future ACL injury risk after ACL reconstruction in soccer athletes from the Multicenter Orthopaedic Outcomes Network (MOON) group. Am J Sports Med. doi:10.1177/0363546512459476

11. Cascio BM, Culp L, Cosgarea AJ (2004) Return to play after anterior cruciate ligament reconstruction. Clin Sports Med 23:395-408

12. Claes S, Verdonk P, Forsyth R, Bellemans J (2011) The "ligamentization" process in anterior cruciate ligament reconstruction: what happens to the human graft? A systematic review of the literature. Am J Sports Med 39:2476-2483

13. Dunn WR, Spindler KP, MOON Consortium (2010) Predictors of activity level 2 years after anterior cruciate ligament reconstruction (ACLR): a Multicenter Orthopaedic Outcomes Network (MOON) ACLR cohort study. Am J Sports Med 38:2040-2050

14. Eriksson K, Anderberg P, Hamberg P, Löfgren AC, Bredenberg M, Westman I et al (2001) A comparison of quadruple semitendinosus and patellar tendon grafts in reconstruction of the anterior cruciate ligament. J Bone Joint Surg Br 83:348-354

15. Feller JA, Webster KE (2003) A randomized comparison of patellar tendon and hamstring tendon anterior cruciate ligament reconstruction. Am J Sports Med 31:564-573

16. Fink C, Hoser C, Hackl W, Navarro RA, Benedetto KP (2001) Long-term outcome of operative or nonoperative treatment of anterior cruciate ligament rupture-is sports activity a determining variable? Int J Sports Med 22:304-309

17. Fujimoto E, Sumen Y, Urabe Y, Deie M, Murakami Y, Adachi N et al (2004) An early return to vigorous activity may destabilize anterior cruciate ligaments reconstructed with hamstring grafts. Arch Phys Med Rehabil 85:298-302

18. Glasgow SG, Gabriel JP, Sapega AA, Glasgow MT, Torg JS (1993) The effect of early versus late return to vigorous activities on the outcome of anterior cruciate ligament reconstruction. Am J Sports Med 21:243-248

19. Gobbi A, Francisco R (2006) Factors affecting return to sports after anterior cruciate ligament reconstruction with patellar tendon and hamstring graft: a prospective clinical investigation. Knee Surg Sports Traumatol Arthrosc 14:1021-1028

20. Hui C, Salmon LJ, Kok A, Maeno S, Linklater J, Pinczewski LA (2011) Fifteen-year outcome of endoscopic anterior cruciate ligament reconstruction with patellar tendon autograft for "isolated" anterior cruciate ligament tear. Am J Sports Med 39:89-98

21. Krych AJ, Jackson JD, Hoskin TL, Dahm DL (2008) A meta-analysis of patellar tendon autograft versus patellar tendon allograft in anterior cruciate ligament reconstruction. Arthroscopy 24:292-298

22. Kvist J (2004) Rehabilitation following anterior cruciate ligament injury: current recommendations for sports participation. Sports Med 34:269-280

23. Kvist J, Ek A, Sporrstedt K, Good L (2005) Fear of re-injury: a hindrance for returning to sports after anterior cruciate ligament reconstruction. Knee Surg Sports Traumatol Arthrosc 13:393-397

24. Langford JL, Webster KE, Feller JA (2009) A prospective longitudinal study to assess psychological changes following anterior cruciate ligament reconstruction surgery. Br J Sports Med 43:377-378

25. Lee DYH, Karim SA, Chang HC (2008) Return to sports after anterior cruciate ligament reconstruction - a review of patients with minimum 5-year follow-up. Ann Acad Med Singapore 37:273-278 
26. Li X, Xu CP, Song JQ, Jiang N, Yu B (2012) Single-bundle versus double-bundle anterior cruciate ligament reconstruction: an up-todate meta-analysis. Int Orthop. doi:10.1007/s00264-12-1651-1

27. Lohmander LS, Ostenberg A, Englund M, Roos H (2004) High prevalence of knee osteoarthritis, pain, and functional limitations in female soccer players twelve years after anterior cruciate ligament injury. Arthritis Rheum 50:3145-3152

28. MacDonald PB, Hedden D, Pacin O, Huebert D (1995) Effects of an accelerated rehabilitation program after anterior cruciate ligament reconstruction with combined semitendinosus-gracilis autograft and a ligament augmentation device. Am J Sports Med 23:588-592

29. Magnussen RA, Carey JL, Spindler KP (2011) Does autograft choice determine intermediate-term outcome of ACL reconstruction? Knee Surg Sports Traumatol Arthrosc 19:462-472

30. Marcacci M, Zaffagnini S, Iacono F, Neri MP, Loreti I, Petitto A (1998) Arthroscopic intra- and extra-articular anterior cruciate ligament reconstruction with gracilis and semitendinosus tendons. Knee Surg Sports Traumatol Arthrosc 6:68-75

31. Mascarenhas R, Tranovich MJ, Kropf EJ, Fu FH, Harner CD (2012) Bone-patellar tendon-bone autograft versus hamstring autograft anterior cruciate ligament reconstruction in the young athlete: a retrospective matched analysis with 2-10 year follow-up. Knee Surg Sports Traumatol Arthrosc 20:1520-1527

32. Meredick RB, Vance KJ, Appleby D, Lubowitz JH (2008) Outcome of single-bundle versus double-bundle reconstruction of the anterior cruciate ligament: a meta-analysis. Am J Sports Med 36:1414-1421

33. Myer GD, Paterno MV, Ford KR, Quatman CE, Hewett TE (2006) Rehabilitation after anterior cruciate ligament reconstruction: criteria-based progression through the return-to-sport phase. J Orthop Sports Phys Ther 36:385-402

34. Myklebust G, Bahr R (2005) Return to play guidelines after anterior cruciate ligament surgery. Br J Sports Med 39:127-131

35. Myklebust G, Holm I, Maehlum S, Engebretsen L, Bahr R (2003) Clinical, functional, and radiologic outcome in team handball players 6 to 11 years after anterior cruciate ligament injury: a follow-up study. Am J Sports Med 31:981-989

36. Øiestad BE, Engebretsen L, Storheim K, Risberg MA (2009) Knee osteoarthritis after anterior cruciate ligament injury: a systematic review. Am J Sports Med 37:1434-1443

37. Pinczewski LA, Lyman J, Salmon LJ, Russell VJ, Roe J, Linklater J (2007) A 10-year comparison of anterior cruciate ligament reconstructions with hamstring tendon and patellar tendon autograft: a controlled, prospective trial. Am J Sports Med 35:564-574

38. von Porat A, Roos EM, Roos H (2004) High prevalence of osteoarthritis 14 years after an anterior cruciate ligament tear in male soccer players: a study of radiographic and patient relevant outcomes. Ann Rheum Dis 63:269-273

39. Scheffler SU, Unterhauser FN, Weiler A (2008) Graft remodeling and ligamentization after cruciate ligament reconstruction. Knee Surg Sports Traumatol Arthrosc 16:834-842
40. Shelbourne KD, Gray T (1997) Anterior cruciate ligament reconstruction with autogenous patellar tendon graft followed by accelerated rehabilitation. A two- to nine-year followup. Am J Sports Med 25:786-795

41. Shelbourne KD, Nitz P (1990) Accelerated rehabilitation after anterior cruciate ligament reconstruction. Am J Sports Med 18:292-299

42. Shelbourne KD, Gray T, Haro M (2009) Incidence of subsequent injury to either knee within 5 years after anterior cruciate ligament reconstruction with patellar tendon autograft. Am J Sports Med 37:246-251

43. Smith FW, Rosenlund EA, Aune AK, MacLean JA, Hillis SW (2004) Subjective functional assessments and the return to competitive sport after anterior cruciate ligament reconstruction. Br J Sports Med 38:279-284

44. Struewer J, Ziring E, Frangen TM, Efe T, Meißner S, Buecking B et al (2012) Clinical outcome and prevalence of osteoarthritis after isolated anterior cruciate ligament reconstruction using hamstring graft: follow-up after two and ten years. Int Orthop. doi:10.1007/ s00264-012-1653-Z

45. Tegner Y, Lysholm J (1985) Rating systems in the evaluation of knee ligament injuries. Clin Orthop Relat Res 198:43-49

46. Waldén M, Hägglund M, Magnusson H, Ekstrand J (2011) Anterior cruciate ligament injury in elite football: a prospective three-cohort study. Knee Surg Sports Traumatol Arthrosc 19:11-19

47. Warner SJ, Smith MV, Wright RW, Matava MJ, Brophy RH (2011) Sport-specific outcomes after anterior cruciate ligament reconstruction. Arthroscopy 27:1129-1134

48. Webb JM, Corry IS, Clingeleffer AJ, Pinczewski LA (1998) Endoscopic reconstruction for isolated anterior cruciate ligament rupture. J Bone Joint Surg Br 80:288-294

49. Webster KE, Feller JA, Lambros C (2008) Development and preliminary validation of a scale to measure the psychological impact of returning to sport following anterior cruciate ligament reconstruction surgery. Phys Ther Sport 9:9-15

50. Wiger P, Brandsson S, Kartus J, Eriksson BI, Karlsson J (1999) A comparison of results after arthroscopic anterior cruciate ligament reconstruction in female and male competitive athletes. A two- to five-year follow-up of 429 patients. Scand J Med Sci Sports 9:290-295

51. Wright RW, Magnussen RA, Dunn WR, Spindler KP (2011) Ipsilateral graft and contralateral ACL rupture at five years or more following ACL reconstruction: a systematic review. J Bone Joint Surg Am 93:1159-1165

52. Zaffagnini S, Bruni D, Russo A, Takazawa Y, Lo Presti M, Giordano G et al (2008) ST/G ACL reconstruction: double strand plus extra-articular sling vs double bundle, randomized study at 3-year follow-up. Scand J Med Sci Sports 18:573-581

53. Zhu Y, Tang RK, Zhao P, Zhu SS, Li YG, Li JB (2012) Doublebundle reconstruction results in superior clinical outcome than single-bundle reconstruction. Knee Surg Sports Traumatol Arthrosc. doi:10.1007/s00167-012-2073-8 